Linguistique, littérature, didactique

183-184 | 2019

oralité, littératie

\title{
Parler, écrire : « continuum communicatif » et rupture matérielle
}

\section{Rudolf Mahrer}

\section{(2) OpenEdition \\ 1 Journals}

\section{Édition électronique}

URL : http://journals.openedition.org/pratiques/6842

DOI : $10.4000 /$ pratiques. 6842

ISSN : 2425-2042

Éditeur

Centre de recherche sur les médiations (CREM)

\section{Référence électronique}

Rudolf Mahrer, «Parler, écrire : « continuum communicatif » et rupture matérielle », Pratiques [En ligne], 183-184 | 2019, mis en ligne le 30 décembre 2019, consulté le 10 décembre 2020. URL : http:// journals.openedition.org/pratiques/6842 ; DOI : https://doi.org/10.4000/pratiques.6842

Ce document a été généré automatiquement le 10 décembre 2020.

(c) Tous droits réservés 


\title{
Parler, écrire : « continuum communicatif » et rupture matérielle
}

\author{
Rudolf Mahrer
}

«D'abord nous parlons : c'est un premier

système.

Nous lisons et écrivons : c'est un système distinct,

spécifique.»

(Benveniste, 2012, p. 61)

1 La façon dont les linguistes pensent le rapport entre l'oralité et la scripturalité (formes de discours, systèmes de signes, média, substances...) est un formidable révélateur de leurs présupposés disciplinaires : leur point de vue théorique, leurs observables, leur conception du langage. Pour contribuer au volet épistémologique du numéro, je commencerai par présenter l'apport de P. Koch et W. Oesterreicher (2001) à la problématique de l'articulation conceptuelle délicate entre l'écrit et l'oral. Le succès rencontré actuellement en France par le travail de ces deux romanistes alimente le courant continuiste qui domine actuellement les débats. Dans un second temps, j'essaierai d'apporter deux compléments au modèle dans sa formulation de 2001. D'abord en levant une indétermination concernant le point de vue adopté (épilinguistique ou métalinguistique); ensuite en rappelant que le cadre proposé marginalise les déterminations matérielles qui pèsent sur la langue et les discours. Or la matérialité des signes détermine l'usage et le développement des systèmes; elle est donc un facteur de discontinuité au sein des idiomes qui à la fois se parlent et s'écrivent.

\section{P. Koch et W. Oesterreicher 2001}

2 Depuis les années 1990, le travail de W. Oesterreicher (1942-2015) et de P. Koch (1951-2014) ${ }^{1}$ constitue une référence incontournable, en Allemagne d'abord, puis 
rapidement dans toute l'Europe. Il est aujourd'hui un passage quasi obligé des approches intégrant à la description des langues celle de leurs réalisations discursives (sociolinguistique, linguistique variationnelle, linguistique de corpus, linguistique textuelle ou analyse conversationnelle). Formateurs de renom - W. Oesterreicher à Tubingue, $P$. Koch à Munich, pour ne mentionner que les universités où ils terminèrent leur carrière, tous les deux brutalement -, auteurs d'une œuvre personnelle abondante, publiant en plusieurs langues romanes et à propos de plusieurs langues romanes, les deux linguistes ont encore en commun de s'inscrire dans le sillage d'E. Coseriu et d'interroger les propriétés linguistiques internes (la thèse de P. Koch portait sur la valence verbale en français, par exemple) compte tenu de facteurs externes: les traditions discursives d'une part, les déterminations cognitives, situationnelles, sociales et médiatiques des discours, d'autre part.

3 À côté de leurs influentes œuvres individuelles, les deux linguistes ont développé une réflexion commune sur la variation linguistique. Mon ambition n'est pas de présenter leur modèle dans son détail et ses évolutions; je me concentrerai sur l'article "Gesprochene Sprache und geschriebene Sprache. Langage parlé, langage écrit", publié en français dans le Lexicon der Romanistischen Linguistik (Koch \& Oesterreicher, 2001, p. 584-618); il s'agira d'avoir cette décontextualisation bien à l'esprit au moment de la discuter ${ }^{2}$. Néanmoins la réduction se justifie : c'est essentiellement à travers cette longue contribution encyclopédique qu'est connu en France le travail des deux romanistes $^{3}$ et les formulations qu'on y rencontre servent d'appui à un grand nombre de recherches actuelles sur corpus.

4 L'article en question concerne les rapports entre oralité et scripturalité ; le "cadre théorique " proposé, excédant cette seule problématique, vise à "présenter la complexité du problème dans une perspective systématique» (ibid., p. 584). On en résumera la teneur comme suit. La variation linguistique, en diachronie comme en synchronie, trouve son premier fondement dans la variété des comportements sociaux ; or ceux-ci sont polarisés: le "domaine de la distance» et le "domaine de la proximité » en forment les deux extrémités. Les variétés du discours s'inscrivent et s'organisent, entre les deux, le long d'un continuum de formes appelé «continuum communicatif $»$.

[1] Le continuum communicatif se définit, en dernière analyse, par des données anthropologiques qui sont à la base de toute communication humaine (ibid., p. 586).

5 Le domaine de la proximité a son langage, «die Sprache der Nähe »" Il répond au «données anthropologiques" suivantes: le besoin d'interagir avec des congénères présents (à la fois ici et maintenant) souvent connus de l'énonciateur et partageant avec lui une même situation, où l'émotion est souvent présente, dans un cadre discursif et un contexte culturel inférables de données partagées. Quant au langage caractéristique du domaine de la distance (" die Sprache der Distanz») ? Il répond à la nécessité d'échanger, "la tête froide", avec des interlocuteurs absents (futurs ou ailleurs), souvent inconnus, et suppose donc une autonomie sémantique et une cohérence textuelle supérieures, ayant à comporter lui-même les indications situationnelles et contextuelles nécessaires à l'effectuation de son projet communicatif. Les déterminations qui pèsent et font varier nos manières de communiquer, on le voit par ce bref résumé, ne font pas intervenir l'opposition entre oralité et scripturalité.

6 Cette opposition entre impératifs communicationnels trouve une manifestation linguistique (au sens étroit) dans les situations de diglossie, comme celle de l'Europe 
médiévale, ou celle de la Suisse allemande contemporaine, dans laquelle une langue est utilisée pour les pratiques discursives de la proximité - les langues vernaculaires, le Suisse allemand -, une autre pour le domaine de la distance - le latin, le Hochdeutsch (ibid., p. 608).

7 Si l'hypothèse de $\mathrm{P}$. Koch et W. Oesterreicher a servi à repenser les rapports entre oralité et scripturalité, c'est qu'elle avait ce problème en point de mire dès l'origine; le titre du premier article que les deux linguistes ont consacré à cette question l'indique : «Sprache der Nähe - Sprache der Distanz. Mündlichkeit und Schriftlichkeit im Spannungsfeld von Sprachtheorie und Sprachgeschichte " «Oralité et scripturalité dans le champ des théories et de l'histoire des langues ", Koch \& Oesterreicher, 1985). Leur contribution au Lexicon der Romanistischen Linguistik s'inscrit dans cette même ligne.

[2] «L'oral et l'écrit ", «l'oralité et la scripturalité », voilà un des domaines privilégiés de la recherche actuelle non seulement en linguistique, mais aussi dans les sciences humaines et sociales en général. (Koch \& Oesterreicher, 2001, p. 584)

Qu'est-ce qui explique la popularité de ce cadre théorique en France? Son grand raffinement, la variété des phénomènes linguistiques envisagés, l'abondance de la documentation dans différentes langues, ou encore la lumière qu'il jette sur les phénomènes diachroniques (ibid., p. 612-614) ? Incontestablement. Sans doute aussi le fait que le français est particulièrement affecté par la variation entre ses réalisations phoniques et graphiques:

[3] [...] si l'italien, le portugais et le roumain offrent une quantité moyenne de différences entre le parlé et l'écrit, le français, quant à lui, exploite massivement cette dimension, tandis que l'espagnol ne la fonctionnalise pratiquement pas. (ibid., p. 607)

9 Acuité du problème qui tombe fort mal, puisque, sur cette question, « la recherche s'est souvent discréditée, même au niveau théorique, par une imprécision d'idée surprenante » (ibid., p. 585), et en particulier «les structuralistes français d'aprèsguerre »: "Obligés de faire face aux phénomènes conceptionnels particulièrement saillants du français parlé, ils les englobent dans un concept flou et ambigu d'oral et d'écrit (cf. Dubois, 1967) »(Koch \& Oesterreicher, 2001, p. 589). Seul Martinet est épargné.

Mais que sont ces «phénomènes conceptionnels particulièrement saillants du français parlé » ? C'est bien par là qu'il faut commencer. Le geste théorique initial de l'article consiste - en s'appuyant sur les observations de O. Behaghel, B. Schlieben-Lange, A. Martinet, T. de Mauro, W. Chafe et enfin L. Söll - à distinguer le «média » du discours de sa " conception ", c'est-à-dire son " allure linguistique ».

[4] Il faut, effectivement, insister sur le fait que la réalisation médiale, phonique ou graphique, est, en principe, indépendante de l'« allure linguistique » de l'énoncé. C'est à ce dernier aspect conceptionnel que L. Söll applique les termes de langue parlée et de langue écrite. (ibid., p. 585)

11 Tenir séparément, autrement dit, la substance des énoncés d'un côté, leur style de l'autre - si par style on entend les propriétés linguistiques et textuelles caractéristiques d'une famille d'énoncés. Reprenant les termes de L. Söll, P. Koch et W. Oesterreicher distinguent fermement deux couples :

1. « code graphique " vs « code phonique » pour penser l'opposition " médiale », qui est une dichotomie entre matérialités différentes ; 
2. « langue parlée » vs " langue écrite » pour appréhender la dimension " conceptionnelle », qui correspond à une opposition entre styles d'énoncés.

12 Cette disjonction permet de distinguer précisément des pratiques métadiscursives qu'on risque de confondre si on les envisage au moyen de la notion vague de passage à l'écrit. La transcription d'une conversation (passage d'un discours de son « code phonique » d'origine au « code graphique », mais gardant son allure " parlée ») ou la lecture à voix haute de cet article (discours de «code graphique " passé au "code phonique " mais demeurant dans son style natif «écrit») sont des processus strictement «transmédiaux ». Au " passage à l'écrit » de la transcription (=Verschriftung), il faut opposer un autre phénomène, "conceptionnel» (stylistique) cette fois : celui de la reformulation d'un énoncé adoptant les formes stylistiques caractéristiques des discours oraux en un autre énoncé adoptant l'allure des discours écrits (=Verschriftlichung). C'est le cas du procès-verbal, qui suppose non seulement un changement de média, mais aussi, une mise aux « normes scripturaires » régulière d'un énoncé adoptant à l'origine les «normes locutoires» (Vachek, 1939) - un passage du parlé à l'écrit, dans les termes de L. Söll repris par P. Koch et W. Oesterreicher.

13 L'originalité du modèle ne réside pas dans la distinction des plans médial et conceptionnel ; jusque-là, P. Koch et W. Oesterreicher sont fidèles à L. Söll. Leur apport propre se situe dans l'hypothèse introduite plus haut et dont on peut dégager trois aspects.

14 (1) Approfondissant la dimension conceptionnelle, P. Koch et W. Oesterreicher postulent que la variation linguisitque n'est pas contrainte par la différence de médium, mais " motivée » par les nécessités communicatives distinctes que réclame la sphère sociale. De manière universelle, la société exigerait des modalités de communication différentes et ces exigences sont le principal facteur de la variation des discours, comme mise en action de la langue.

15 (2) Les exigences universelles de la communication sont polarisées. Les déterminations socio-cognitives qui pèsent sur les énoncés s'opposent et constituent deux extrêmes opposés : le « domaine de l'immédiat » (illustré par la communication entre amis) et le «domaine de la distance» (illustré par le texte de loi). À chacun de ces pôles correspondent des "stratégies » de communication également opposées : le "langage de la distance », ou langue écrite, et le «langage de la proximité », ou langue parlée. Entre ces deux pôles se déploie le "continuum communicatif», soit l'ensemble des traditions discursives d'une langue.

[5] Au niveau universel ${ }^{5}$, la problématique du parlé et de l'écrit se présente sous la forme de décalages caractéristiques dans l'activité langagière : quelle que soit leur langue, les sujets parlants répondent dans leurs actes de langage aux exigences de l'immédiat et de la distance par des stratégies communicatives, concernant la référenciation, la prédication, la contextualisation, l'orientation spatio-temporelle, etc. Les stratégies communicatives étant déterminées par des facteurs cognitifs fondamentaux, tous ces phénomènes ont un statut universel. (Koch \& Oesterreicher, 2001, p. 588)

16 Ces deux pôles correspondent à des extrêmes de notre «comportement communicatif »: s'y observent autrement dit des «stratégies communicatives » opposables, en tant qu'elles répondent «aux conditions communicatives universelles 
de l'immédiat et de la distance » (ibid., p. 591). L'universalité en question est qualifiée de «fonctionnelle» au sens où les structures linguistiques stimulées par les « impératifs communicatifs » peuvent varier de langue en langue, elles sont néanmoins apparentables par leur fonction commune (ibid., p. 592) : par exemple l'hypotaxe, dont les deux romanistes expliquent bien en quoi elle répond aux besoins de la distance, prend des formes variables en anglais et en italien.

17 (3) Sur la base des travaux (principalement pragmatiques, psycholinguistiques et sociolinguistiques; ibid., p.586) concernant l'interaction de la langue avec ses extériorités, P. Koch et W. Oesterreicher analysent l'opposition des comportements communicatifs en dix facteurs. Ceux-ci sont les paramètres permettant de situer les genres du discours dans le continuum de la communication (ibid., p. 601).

" Paramètres pour caractériser le comportement communicatif des interlocuteurs par rapport aux déterminants situationnels et contextuels » (ibid., p. 586)

\begin{tabular}{|l|r|}
\hline 1) communication privée & communication publique (1 \\
\hline 2) interlocuteur intime & interlocuteur inconnu (2 \\
\hline 3) émotionnalité forte & émotionnalité faible (3 \\
\hline 4) ancrage actionnel et situationnel & détachement actionnel et situationnel (4 \\
\hline 5) ancrage référentiel dans la situation & détachement référentiel de la situation (5 \\
\hline 6) coprésence spatio-temporelle & séparation spatio-temporelle (6 \\
\hline 7) coopération communicative intense & coopération communicative minime (7 \\
\hline 8) dialogue & monologue (8 \\
\hline 9) communication spontanée & communication préparée (9 \\
\hline 10) liberté thématique & fixation thématique (10 \\
\hline etc. & etc. \\
\hline
\end{tabular}

18 La position d'une tradition discursive dans le continuum, selon la combinaison d'ingrédients qu'elle comporte, va déterminer une attente "conceptionnelle» (stylistique) ; elle va, par suite, conditionner la sensibilité aux variations diastratiques, diaphasique et diatopiques. Autrement dit, l'appréciation de la variation est relative à la position du discours considéré sur le continuum communicatif. Par exemple le mot chiotte (dans la série automobile - voiture - bagnole - chiotte; ibid., p. 605) sera jugé populaire dans la langue parlée et vulgaire dans la langue écrite. De manière analogue, une forme n'est jugée dialectale que dans la mesure où elle n'appartient qu'aux comportements de la proximité. $\mathrm{P}$. Koch et $\mathrm{W}$. Oesterreicher apporte ainsi une réponse forte et originale à la question de l'architecture des langues : c'est le «continuum communicationnel » qui organise leur « profil variationnel » (ibid., p. 605).

[6] En dernière analyse, on est amené à constater que c'est le continnum conceptionnel qui détermine l'organisation de l'espace variationnel tout entier et

l'emploi de tout moyen d'expression linguistique, qu'il soit marqué dans la dimension diatopique, diastratique ou diaphasique. (ibid., p. 605)

19 En conclusion, pour les deux romanistes, le phénomène universel, c'est-à-dire translinguistique, qui structure la variation discursive, n'a pas un soubassement 
médiatique. Ce qui conditionne en dernier ressort les formes de l'énonciation, et par conséquent le profil variationnel d'une langue, ce sont les paramètres communicatifs polarisés "form[ant] le cadre de toutes les pratiques communicatives historiques " (ibid., p. 601). En somme, le parlé et l'écrit sont pour eux le nom des stratégies opposées répondant aux déterminations contextuelles, situationnelles et cognitives de la distance et de l'immédiat.

\section{Propositions de compléments}

\subsection{Points de vue métalinguistique et épilinguistique}

Pour défendre l'autonomie de la polarisation des pratiques communicationnelles relativement à l'opposition médiale, P. Koch et W. Oesterreicher font notamment l'observation suivante: dans les civilisations sans écriture, les «impératifs communicatifs » de la distance se manifestent également ; épopée orale, proverbe ou incantations en constituent quelques illustrations (ibid., p. 587). Toute société, même sans écriture, réclame des pratiques distales. Partons de cet argument convaincant pour décrire deux aspects du modèle qui me semblent inviter à des compléments.

21 Dès lors qu'est démontrée l'autonomie du continuum communicatif, dont le fondement est essentiellement social, relativement à l'opposition phonique/graphique qui est, quant à elle, d'ordre médial, pourquoi continuer à utiliser les termes d'écrit et de parlé pour désigner les extrêmes conceptionnels? Pourquoi conserver la typologique de L. Söll et ne pas aller jusqu'au bout de la logique d'autonomisation des plans en systématisant une opposition entre "discours de la distance " et "discours de l'immédiat» (qui existe d'ailleurs dans l'article et concurrence l'opposition entre « langue écrite » et « langue orale ») ?

Dans le contexte de la clarification que doivent apporter P. Koch et W. Oesterreicher sur les relations entre oralité et écriture, on comprend que la terminologie de L. Söll constitue une manière de dialoguer avec la tradition et le sens commun ${ }^{6}$. Pourtant, même après avoir contractualisé les termes, n'est-ce pas replonger dans l'obscurité qu'on voulait dissiper que d'utiliser le terme "écrit ", si étroitement associé au champ sémantique de la scripturalité, pour désigner des discours communiqués oralement (le journal télévisé, la plaidoirie, le cours ex cathedra...) et d'autres qui peuvent n'avoir pas même sollicité l'écriture au moment de leur production (le proverbe, l'épopée de tradition orale) ? Ce choix terminologique gaspille une partie du bénéfice de l'analyse. Nous revoilà dans la situation de J. Gardes Tamine qui, voulant cerner l'aire d'usage des appositions, après bien des caractérisations sociales et génériques, concède l'assimilation :

[7] On la trouve surtout dans des discours techniques ou dans « tout ce qui peut être considéré comme "discours public", avec ou sans interlocuteurs: les discours professionnels, les débats et entrevues publics, les émissions de radio ou de télévision. Ce sont, dans l'ensemble, des situations de "protocole", c'est-à-dire des situations d'oral écrit, si l'on peut dire. (Gardes Tamine, 2004, p. 151)

Pour un locuteur francophone, recourir aux variétés distales en parlant, c'est "parler écrit » ou " parler comme un livre »7.

Cet aspect terminologie semble toucher à un enjeu théorique et méthodologique important, celui de l'articulation de deux points de vue différents sur la variation 
linguistique : le point de vue métalinguistique, savant, fondé désormais sur les preuves apportées par P. Koch et W. Oesterreicher que l'analyse du profil variationnel doit se situer non sur le plan des médias, mais sur celui des exigences de la vie sociale, et le point de vue épilinguistique, ordinaire, selon lequel l'oral et l'écrit constituent les catégories spontanées pour décrire les extrémités « conceptionnelles » du français.

Il est juste que la théorie variationnelle critique la tendance spontanée à identifier plan médial et plan conceptionnel ${ }^{8}$ - notamment en rappelant que tout ce qui s'écrit peut se dire et inversement ${ }^{9}$. Mais elle doit également rendre compte de la force et des effets de cette assimilation spontanée. Comme tous les leurres répandus à l'échelle d'une culture, elle atteste une idéologie, et non une erreur de jugement. L'assimilation médiatico-stylistique est une composante essentielle de l'imaginaire langagier des locuteurs dans les sociétés à écriture - et mieux que tout autre, le modèle de P. Koch et W. Oesterreicher permet d'expliquer pourquoi. Or cet imaginaire est à l'œuvre dans un geste interprétatif récurrent: celui qui conduit à considérer comme "parlés" des énoncés pourtant graphiques - ceux de toute une littérature dite justement «parlante » (Meizoz, 2001; Philippe, 2009; Mahrer, 2017) - en vertu de propriétés stylistiques qui sont grosso modo celles de l'immédiat (dialogue, ancrage situationnel, phénomènes de corrections, marqueurs de tour de parole, etc.).

Par ce geste interprétatif (on peut l'appeler ancrage stylistique, voir Mahrer, 2016, p. 14-16), l'interprète identifie la «tradition discursive » d'un énoncé ${ }^{10}$ à la faveur de sa ressemblance "conceptionelle", c'est-à-dire de sa manière de dire. Ce mécanisme participe en profondeur à l'interprétation des textes en orientant des inférences concernant leur énonciateur et leur énonciataire, leurs moyens et leurs fins.

Pour «faire parler » leurs personnages ou leur narrateur, L.-F. Céline, C. F. Ramuz, R. Queneau ou R. Pinget adoptent dans l'écrit romanesque la langue de l'immédiat. Dira-t-on que leur conception de la variation est simpliste parce qu'ils confondent oralité et immédiateté ? Et dira-t-on aux lecteurs qui trouvent ces romans "parlants » qu'en fait ils ne savent pas lire, qu'il n'y a rien d'oral là-dedans ? Il n'y a qu'une variété possible d'un continuum ni oral, ni écrit. La démarche linguistique consiste-t-elle à expliquer au locuteur-lecteur ce qu'il doit considérer comme caractéristique de l'oral et de l'écrit, ou doit-elle observer ce qu'il reconnaît comme tel et expliquer pourquoi ?

En définitive, le maintien des termes de parlé et d'écrit comme descripteurs génériques des styles propres aux extrêmes communicationnels conduit à confondre deux points de vue qu'il est nécessaire de distinguer : le plan métalinguistique (exogène, savant) de la connaissance du linguiste, fondé sur une théorie explicite de la variation, et celui, épilinguistique (indigène, populaire) de la compétence linguistique des locuteurs, à l'œuvre dans leurs processus interprétatifs (Brekle, 1989).

\subsection{Perspective matérialiste : analyse biotechnologique des systèmes de signes}

Si elle marque un pas décisif pour la description du profil variationnel des langues, la position de P. Koch et W. Oesterreicher - dans sa formulation de 2001 du moins marginalise la question de l'influence du medium sur les pratiques. En effet, tout en définissant les "allures des énoncés» relativement aux impératifs sociaux, mais indépendamment des médias, $\mathrm{P}$. Koch et $\mathrm{W}$. Oesterreicher reconnaissent d'évidentes convergences entre ces plans. Associant le développement culturel et les pratiques 
communicatives de la distance, ils relèvent la «stimulation » produite par le média écrit (envisagé, significativement dans ce passage, non plus comme « code graphique ", mais comme « scripturalité médiale ») :

[8] En réalité, c'est la distance communicative qui garantit le plein épanouissement des valeurs sociales, intellectuelles et spirituelles d'une civilisation, et cela même avant l'introduction de l'écriture dans une société. On ne saurait nier, d'autre part, que la scripturalité médiale stimule énormément cet épanouissement culturel dans le domaine de la distance. Il constitue, sans aucun doute, un catalyseur décisif. (Koch \& Oesterreicher, 2001, p. 590)

Ailleurs, ils reconnaissent des « affinités » :

[9] Ceci dit, il faut évidemment reconnaître les affinités qui existent entre le code phonique et la conception parlée d'une part et entre le code graphique et la conception écrite d'autre part. C'est ce qui explique d'ailleurs pourquoi on n'a pas hésité à identifier, depuis toujours, le phonique avec le parlé et le graphique avec l'écrit. Mais une telle simplification nous empêcherait, justement de prendre en considération l'éventail tout entier des constellations et options médioconceptionnelles ${ }^{11}$. (ibid., p. 585, 603)

On ne peut qu'être surpris par cette rhétorique de l'évidence (" évidemment ", « sans aucun doute », «bien sûr ») dans un modèle si puissamment explicatif. Ce qui est clair, c'est qu'après avoir, par hypothèse, déconnecté tout à fait média, styles des énoncés et enjeux sociaux, $\mathrm{P}$. Koch et $\mathrm{W}$. Oesterreicher ne peuvent plus guère s'engager au-delà de la stricte observation de l'attraction de ces plans. Dans une première réception du travail des deux romanistes, en 1998, Brigitte Schieben-Lange formulait une critique analogue :

[10] La séparation stricte du médial et du conceptionnel amène les auteurs [P. Koch et $\mathrm{W}$. Oesterreicher] à qualifier les rapports qui existent entre oral (médial) et proximité (conceptionnel), d'un côté, et écrit et distance, d'un autre côté, de simple affinité. À mon avis, ce rapport est beaucoup plus étroit: les contraintes et les possibilités conceptionnelles émanent justement des traits constitutifs du medium. [...] Bien sûr, il ne s'agit pas d'une détermination absolue, mais d'un savoir élocutionnel issu d'une analyse constitutive des conditions médiales de la parole. Si on coupe ce lien, les paramètres des stratégies conceptionnelles deviennent arbitraires. (Schlieben-Lange, 1998, p. 267)

31 Face à la voie qui consiste à autonomiser tout à fait les plans médiatiques, stylistiques (conceptionnelles) et sociaux - puis à s'étonner de leur mystérieuses affinités -, il y a une manière complémentaire qui ne revient pas pour autant à adopter le point de vue assimilateur (voie simplificatrice dont on a vu néanmoins pourquoi on ne pouvait se satisfaire de l'écarter tout à fait). Il est possible en effet de saisir l'affinité du média avec la conception, sans réduire l'éventail des "options médio-conceptionnelles ", c'est-àdire la variété interne aux productions orales et écrites. C'est la voie, matérialiste, que j'aimerais esquisser en conclusion.

Tout en souscrivant à l'hypothèse de P. Koch et W. Oesterreicher, qui me semble de nature anthropologique, et en admettant l'autonomie relative d'un plan essentiel de développement du langage qui relève des impératifs sociaux, la linguistique doit chercher à expliquer pourquoi, par exemple, dans les littératies, l'écrit sert tout particulièrement les fins de la distance (au point de stimuler le développement de ses moyens communicatifs) et l'oralité, les fins de la proximité. On attend d'une approche sémiologique intégrative qu'elle explique pourquoi le média fonctionne comme attracteur pour certains appareils formels et pour certains comportements cognitifs et 
sociaux. Pour répondre à cette question, il faut procéder à ce que Schlieben-Lange appelle " analyse des conditions médiales de la parole».

Remarquons d'abord dans cette perspective que la terminologie "code graphique » vs « code phonique » adoptée pour décrire le plan médial ne convient pas : la notion de code conduit à rater la consistance matérielle du langage. Meilleure que celle de canal, la notion de medium/média reste elle-même imprécise, suggérant que la matérialité des signes n'est qu'un moyen différent pour arriver aux mêmes fins. Pour une approche sémiologique, c'est la nature matérielle des signaux qui est fondatrice et qui doit constituer l'entrée de l'analyse. Affirmant cela, je m'appuie sur le seul essai de sémiologie publié par Benveniste (1974 [1969]) : "Sémiologie de la langue ». Dans cet article, Benveniste insiste sur le fait que la caractérisation d'un système de signes réclame la prise en considération aussi bien des données matérielles et contextuelles que des données formelles, relatives aux signes ou au système lui-même. Sa description des «caractères distinctifs » des systèmes commence d'ailleurs par deux propriétés « empiriques»:

[11] Un système sémiologique se caractérise :

$1^{\circ}$ par son mode opératoire,

$2^{\circ}$ par son domaine de validité,

$3^{\circ}$ par la nature et le nombre de ses signes,

$4^{\circ}$ par son type de fonctionnement.

Le MODE OPÉRATOIRE est la manière dont le système agit, notamment le sens (vue,

ouie, etc.) auquel il s'adresse. (Benveniste, 1974 [1969], p. 51-52)

Toute pratique symbolique est déterminée par la matérialité qu'informent ses signes, les organes et éventuellement les outils que leur production et leur perception requièrent. Ces déterminations, à la fois biologiques et technologiques, conditionnent l'activité individuelle, les pratiques socio-discursives (les domaines de validité du système et les fonctions sociales qu'il permet d'accomplir) et le système lui-même dans sa structure sémiotique. L'analyse biotechnologique est avant tout un problème de substance : elle questionne la manière dont la matérialité des signes conditionne leur utilisation (production, révision, communication). Mais ce conditionnement n'est pas de l'ordre de la causalité. Par exemple, à propos de ce que l'on appelle aujourd'hui disfluence (Shriberg, 2001), phénomène réputé si caractéristique de l'oral, les deux romanistes ne manquent pas d'observer que dans les genres oraux de la distance (ils mentionnent le serment, la prière, le discours funèbre, le prononcé d'une sentence et la visite guidée), les traces du travail de production n'apparaissent pas ou presque pas. Ils peuvent ainsi conclure :

[12] la réalisation phonique d'un discours n'est pas une condition suffisante pour qu'apparaissent sans distinction tous les phénomènes d'hésitation et de correction : en fin de compte, ce sont des contraintes conceptionnelles qui font disparaître ces phénomènes. (Koch \& Oesterreicher, 2001, p. 594)

Et ils ont incontestablement raison: on ne trouvera pas, dans la matérialité des systèmes de signes, des «conditions suffisantes" expliquant tel comportement communicatif, telle variété stylistique. Néanmoins la genèse des systèmes de signes et des traditions discursives soulève cette question : pourquoi tel média a été préféré pour effectuer telle action dans l'espace sociale ? Pourquoi en l'occurrence tous les genres choisis par P. Koch et W. Oesterreicher pour illustrer l'absence, dans certains discours oraux, du travail de production, sont des genres certes communiqués par oral, mais 
ordinairement préparés par écrit? Voilà qui revient à se demander pourquoi l'écrit favorise la disparition des disfluences.

L'analyse biotechnologique des systèmes de signes offre ici un complément nécessaire. Elle relèvera en l'espèce que l'écrit est un système à traces alors que l'oral est un système à processus (Mahrer, 2017, p. 53-55), clivage profond parmi les systèmes de signes. Pourvue d'une rémanence relative, la trace survit à son émission, dispose à la réévaluation de l'énoncé par son producteur après émission, et à sa révision : elle se prête particulièrement bien à un processus d'élaboration cumulatif. Le signal phonique quant à lui, successif et sans support, doit être reproduit et donc mémorisé au moins partiellement pour être révisé, ce qui complique l'opération.

Bien sûr, la rémanence du signal graphique n'est pas une condition suffisante de son perfectionnement: tout écrit n'a d'ailleurs pas vocation à être réécrit et il est plutôt rare qu'on mette ses listes de courses au net. Inversement, en dépit de la charge importante que suppose la gestion en temps réel des multiples dimensions de la production d'un énoncé oral en situation, un orateur aguerri saura faire disparaître, même lorsqu'il improvise, les traces du processus de formulation. Il n'en demeure pas moins que le signal graphique dispose l'énonciateur à la réévaluation et à la révision et qu'il se prête ainsi idéalement à la préparation d'une communication à venir - dans les cas où l'intention de communication et la situation peuvent être anticipées. Le signal acoustique, qui ne suppose aucune technologie et peut être émis et communiqué presque exactement dans le temps de sa production, dispose admirablement bien l'orateur à s'adapter aux variations imprévues de l'interaction (nouvelle question, nouvel interlocuteur, changement de cadre discursif ou de thématique, changement de projet communicatif...). À l'âge de l'éloquence, il n'est pas rare de trouver des jugements sévères à l'encontre de l'écriture, susceptible d'affaiblir cette faculté d'ajustement qui caractérise le bon orateur ${ }^{12}$. En définitive, les médias ne sont pas des conditions suffisantes des comportements communicatifs, mais ils ont des propriétés matérielles qui inclinent à certains usages. Ils ont ce qu'on appelle une "affordance " (Gibson, 1979).

C'est à la faveur de son affordance - la façon dont il dispose à son usage - que l'écrit profite à l'épanouissement de la distance en permettant, pour s'en tenir à l'aspect évoqué, l'élimination, dans les situations sociales où cela est profitable, de tout ce qui semble nuisible à l'action visée par le discours, et l'ajout de tout ce qui peut assurer son effectuation. Une analyse du média - à affiner en fonction des différentes technologies d'écriture, du calame au smartphone, qui modulent leur affordance - complète l'approche de P. Koch et W. Oesterreicher en expliquant pourquoi l'oralité et la scripturalité servent et stimulent les impératifs communicationnels de la proximité et de la distance, respectivement.

\section{Conclusion}

Pourquoi le français offre-t-il une gamme de formulations possibles entre «Je ne sais pas » et «Chépa », entre « Ma bicyclette a disparu!» et « Mon vélo!», entre «Depuis deux ans, il m'arrive de vendre par exemple des salades ou des épinards» et « Moi, ça fait deux ans que j'en vends des salades, des épinards ou comme ça »? P. Koch et W. Oesterreicher répondent à cette question - celle de la variation linguistique - en organisant l'ensemble des interactions sociales entre deux pôles se caractérisant par 
des impératifs sociaux opposés et déterminant des comportements communicatifs distincts. C'est la polarité de ces exigences sociales qui permettrait d'arpenter l'écart variationnel maximal d'une langue selon des différences de «stratégies communicatives concernant la référentiation, la prédication, la contextualisation, l'orientation spatio-temporelle, etc.» (Koch \& Oesterreicher, 2001, p. 588). Voilà l'hypothèse qui confère au modèle proposé une portée transhistorique et translinguistique.

Qu'en ressort-il concernant la distinction entre langue orale et langue écrite? La tendance actuelle consiste à postuler qu'il s'agit d'un même système (voir note 10) empruntant deux médias, ceux-ci pesant sur la variation diaphasique au titre de paramètre contextuel ou générique. Si P. Koch et W. Oesterreicher (2001) n'adoptent pas cette option, leur modèle contribue également à marginaliser le rôle du médium au point de ne pas même compter celui-ci parmi les paramètres qui déterminent la « conception» des discours ${ }^{13}$.

41 Un point de vue matérialiste sur le langage conduit au contraire à reconnaître les substances phonique et graphique comme un facteur fort de discontinuité : leurs affordances très distinctes inclinent la langue orale et la langue écrite à remplir des fonctions sociales différentes et même à se développer en deux systèmes partiellement différents. Qu'il soit de la distance ou de la proximité, le discours écrit doit composer sans l'apport de la prosodie et de la mimogestualité, mais avec la partie signifiante de son espace graphique (Anis, 1988), strate propre à l'écrit dont la contribution au sens est spécifique. Ce fait seul - qui n'est pas seulement médiatique, mais aussi sémiologique - ne manque pas d'affecter la « conception » des discours écrits.

S'il existe indéniablement des facteurs assimilateurs (en particulier la syntaxe et la textualité), la prise en compte de la matérialité des signes problématise la conception continuiste de l'oral et de l'écrit. Le point de vue matérialiste introduit les éléments nécessaires à la compréhension de ce que la scripturalité fait à l'homme, à la société et à la langue elle-même ; autrement dit, il permet d'appréhender l'épanouissement de la distance socio-communicationnelle dans et par l'écrit, et encore de poser le problème difficile de l'identité d'un idiome réunissant deux systèmes radicalement hétérogènes sur le plan de leur substance, ou enfin d'interroger l'effet de cette hétérogénéité sur la cognition. En somme, sans prendre en considération ce que Benveniste appelait le «mode opératoire» des systèmes de signes, il ne sera pas permis au linguiste de nourrir « l'espoir de pousser plus loin l'analyse des effets de l'écriture sur les "modes de pensées" (ou les processus cognitifs) d'une part et sur les institutions sociales les plus importantes d'autre part » (Goody, 1979 [1977], p. 31). C'est pourquoi le point de vue de P. Koch et W. Oesterreicher, essentiel, doit être complété par un autre, complémentaire, selon lequel la condition de l'homme dans le langage ne peut être questionnée abstraction faite de la matérialité des signes. 


\section{BIBLIOGRAPHIE}

ANIS, J. (1988). L'écriture. Théories et descriptions. Paris/Bruxelles : Éditions universitaires/De Boeck. BENVENISTE, É. (1974 [1969]). « Sémiologie de la langue ». Problèmes de linguistique générale 2. Paris : Gallimard, p. 43-66.

BENVENISTE, É. (2012). Dernières leçons. Collège de France, 1968 et 1969. Paris : Éditions de l'EHESS/ Gallimard/Éditions du Seuil.

BLANCHE-BENVENISTE, C. \& JEANJEAN, C. (1987). Le Français parlé. Transcription et édition. Paris : Éditions Didier.

BREKLE, H. E. (1989) : « La linguistique populaire ». In : Auroux S. (dir.). Histoire des idées linguistiques. Tome 1 : la naissance des métalangages en Orient et en Occident, Liège/Bruxelles :

P. Mardaga, p. 39-44.

COSERIU, E. (1983). Introducción a la lingüística. Madrid : Gredos.

DUBOIS, J. (1967). Grammaire structurale du français : le verbe. Paris : Larousse.

GÉvaudan, P. (2014). « Nécrologie. Peter Koch ». Revue de linguistique romane 311-312, t. 78, p. 597-601.

GADET, F. (2017). «L'oralité ordinaire à l'épreuve de la mise en écrit : ce que montre la proximité ». Langages 208, p. 113-126.

GADET, F. \& GUÉRIN E. (2008). « Le couple oral/écrit dans une sociolinguistique à visée didactique ». Le français aujourd'hui 162, p. 21-27.

GARDES TAMINE, J. (2004). Pour une grammaire de l'écrit. Paris : Belin.

GIBSON, J. J. (1979). The Ecological Approach to Visual Perception. Boston : Houghton Mifflin.

GOODY, J. (1979) [1977]. La Raison graphique. La domestication de la pensée sauvage. Trad. de l'anglais par J. Bazin et A. Bensa. Paris : Éditions de Minuit.

KOCH, P. \& OESTERREICHER, W. (1985). « Sprache der Nähe - Sprache der Distanz. Mündlichkeit und Schriftlichkeit im Spannungsfeld von Sprachtheorie und Sprachgeschichte ». Romanistisches Jahrbuch 36, p. 15-43.

KOCH, P. \& OESTERREICHER, W. (1990). Gesprochene Sprache in der Romania : Französisch, Italienisch, Spanisch. Tubingue : Max Niemeyer Verlag.

KOCH, P. \& OESTERREICHER, W. (2001) : « Langage oral et langage écrit ». In : Holtus, G., Metzeltin, M., \& Schmitt, C. (éds), Lexikon der romanistischen Linguistik. Band I, 2: Methodologie. Tubingue : Max Niemeyer Verlag, p. 584-627.

LEBRAVE, J.-L. (1995). «Penser, dicter, écrire : pour une histoire des pratiques de composition ». The Romanic Review 86/3, p. 437-450.

LYONS, J. (1981). Language and Linguistics. An Introduction. Cambridge/New York : Cambridge University Press.

MAHRER, R. (2016). « Du discours indirect libre dans les Annales. Les traductions de Tacite comme observatoire de la compétence langagière $(1790,1830,1925)$ ». In : Philippe G. \& Zufferey J. (dirs), 
Marges et contraintes du discours indirect libre. Fabula/Les colloques. En ligne : http://

www.fabula.org/colloques/document3436.php.

MAHRER, R. (2017). Phonographie. La représentation écrite de l'oral en français. Berlin/Boston : De Gruyter.

MARTINET, A. (1960). Éléments de linguistique générale. Paris : Armand Colin.

MEIZOZ, J. (2001). L'Âge du roman parlant (1919-1939). Écrivains, critiques, linguistes et pédagogues en débat. Genève : Droz.

MODicom, P.-Y. (2015) : « L'opposition oralité - scripturalité dans l'analyse de discours/textes : une introduction au programme de Koch \& Oesterreicher ». Atelier du groupe ÉLIS. Paris : Université Paris-Sorbonne, déc. 2015. En ligne : https://halshs.archives-ouvertes.fr/halshs-01242845v2/ document.

PHILIPPE, G. (2009). « Langue littéraire et langue parlée ». In : Phillipe G. \& Piat J. (dirs), La Langue littéraire. Une histoire de la prose en France de Gustave Flaubert à Claude Simon. Paris : Fayard, p. 57-90. REY-DEBOVE, J. (1988). « À la recherche de la distinction oral/écrit ». In : Catach N. (éd.), Pour une théorie de la langue écrite. Paris : Éditions du CNRS, p. 77-90.

RIEGEL, M., PELLAT, J.-C. \& RIOUL, R. (2009) [1994]. Grammaire méthodique du français. Paris : Presses universitaires de France.

SHRIBERG, E. (2001). « To 'errrr' is human: ecology and acoustics of speech disfluencies ». Journal of the International Phonetic Association 31/1, p. 153-169.

SCHLIEBEN-LANGE, B. (1998). « Les hypercorrections de la scripturalité ». Cahiers de linguistique française 20, p. 255-273.. En ligne : https://clf.unige.ch/files/5514/4103/2706/13-Schlieben.pdf.

VACHEK, J. (1939). «Zum Problem des geschrieben Sprache ». Travaux du cercle linguistique de Prague 8, p. 94-104.

\section{NOTES}

1. Pour une présentation synthétique du travail de P. Koch, voir Gévaudan (2014).

2. Ainsi ma démarche complète celle de P.-Y. Modicom (2015) dont le parti est inverse : résumer l'apport des deux romanistes à partir de leurs principales contributions allemandes, en proposant sa propre traduction terminologique et sans considérer l'article paru en français en 2001.

3. F. Gadet a joué un rôle important dans la diffusion de leur théorie en France. Jusqu'à la très populaire Grammaire méthodique du français qui, depuis sa réédition de 2009, intègre la réflexion des deux romanistes dans leur section "La distinction oral/écrit » (Riegel, Pellat \& Rioul, 2009 [1994], p. 52-56), où l'on retrouve le schéma paramétrique $\mathrm{du}$ "continuum communicatif»-un must désormais, que je ne manquerai pas de reproduire à mon tour.

4. Comme Schlieben-Lange (1998) ou Gadet \& Guérin (2008), je traduis « die Nähe » par « proximité » (alors que l'article de 2001 propose « immédiat »).

5. Référence au modèle d'E. Coseriu (1981), qui distingue, dans l'analyse du langage, trois niveaux: universel, historique (celui des traditions discursives et des variétés historiques d'une langue donnée) et individuel. 
6. Assimilation du métadiscours ordinaire qu'on trouve thématisée ainsi, dans une page consacrée à "la mise en place d'une notation graphique" par acculturation: "les sujets parlants identifient le domaine de la langue écrite et, par là même, le médium graphique traditionnellement avec la langue de culture dominante [...]» (Koch \& Oesterreicher, 2001, p. 617, je souligne).

7. «Parler comme un livre» et non «comme une liste de courses »... le livre représentant, dans les littératies, l'écrit de prestige. Le métalangage ordinaire est sensible aux nuances des variations conceptionnelles au sein de l'écrit. Même un énoncé oral peut être caractérisé de brouillon, ce dernier, bien que d'origine graphique, étant le symbole de l'essai d'énonciation inaboutie et chaotique. C'est principalement dans son opposition schématique à l'oral que l'écrit vaut pour le stéréotype de l'énonciation distale.

8. En linguistique française, la dénonciation des "grands mythes séparateurs" par Blanche-Benveniste et Jeanjean (1987) marque un moment important de cette distanciation.

9. Principe du «transcodage médial de tout énoncé » (Koch \& Oesterreicher, 2001, p. 585) : « it is possible to read aloud what is written and, conversely, to write down what is spoken " (Lyons, 1981, cité dans Koch \& Oesterreicher, 2001, p. 585 ; voir aussi pour des remarques analogues Martinet, 1960, p. 160 ; Rey-Debove, 1988, p. 86 ou, plus récemment Gadet, 2017, p. 121). Cette position me semble néanmoins largement sujette à caution. Pour ne considérer le problème que dans le sens écrit > oral, que faut-il entendre ici exactement par "read aloud»? Le fait qu'on puisse lire à haute voix (oraliser) un énoncé écrit signifie-t-il qu'il y ait strict transcodage, à savoir opération sans interprétation ni transformation du texte source lors du passage dans un autre système ? Peut-on lire un homophone hétérographe (« Entre deux mots, il faut choisir le moindre ", Valéry) sans perte d'informations ? Et comment lire une fin de paragraphe, une note de bas de page, une rature... ? La lecture est irréductible à l'actualisation du système phonographique, associant phonèmes et graphèmes (Mahrer, 2017, p. 109-115). L'affirmation abrupte du "transcodage médial de tout énoncé » postule une homologie totale entre langue écrite et langue orale; or celle-ci n'est que partielle sur le plan morphologique ainsi que sur le plan des systèmes paraverbaux (prosodie à l'oral, topographie à l'écrit). Ce postulat d'homologie, insuffisamment questionné, sous-tend les positions continuistes.

10. À la suite d'E. Coseriu, P. Koch et W. Oesterreicher parlent de "traditions discursives" pour "englobe[r] les types de texte, les genres (littéraires et nonlittéraires), les styles, etc.» (Koch \& Oesterreicher, 2001, p 588); par ailleurs ils définissent ces traditions comme des «schémas de production et de réception " (ibid., p. 601).

11. Affinités envisagées également à propos de l'évolution des langues : «Bien entendu, la progression/régression médiale accompagne presque toujours la progression/ régression conceptionnelle d'un idiome » (Koch \& Oesterreicher, 2001, p. 587).

12. Quintilien estimait par exemple qu' 'il arrive que la pensée nous ramène vers ce que nous avons élaboré par écrit et ne nous permet pas de tenter la chance du moment » (Institution oratoire, X, 7 cité dans Lebrave, 1995).

13. Paradoxalement, ils reconnaissent qu'un genre qui passe d'un média à un autre (interview de presse écrite > interview radiodiffusé, par exemple, p.602) devient nécessairement un nouveau genre. Observation incontestable qui atteste de l'effet 
important, et auquel P. Koch et W. Oesterreicher sont très sensibles, du média sur le genre.

\section{RÉSUMÉS}

Suite à la publication en français d'un important article de synthèse, le modèle proposé par P. Koch et W. Oesterreicher (2001) pour penser la relation entre langue orale et langue écrite est très populaire en Europe, en particulier dans le domaine francophone. On y trouve défendue l'idée que la variation linguistique répond à des impératifs sociaux opposés : la "langue écrite » désigne en fait les manières de parler propres aux situations de "distance communicative » (textes de loi, mais aussi conférence ou plaidoirie) et la « langue orale » correspond aux manières de dire utilisées dans les situations de proximité (conversation, courriel à un proche...). Ainsi définie, l'opposition langue écrite/langue orale a peu à voir avec le medium : elle est déterminée socialement. Après un bref résumé de cette position, cette contribution apporte deux éclairages complémentaires, en expliquant pourquoi il est nécessaire, à propos de l'assimilation entre media et style («conception») des énoncés, de distinguer point de vue du linguiste et point de vue de l'usager; et pourquoi l'indéniable pertinence du point de vue social sur la variation linguistique ne doit pas conduire à écarter un autre aspect sémiologique essentiel de la question, à savoir la manière dont les systèmes de signes et leur emploi sont conditionnés par leur substance (graphique ou phonique).

Since the 1980s, P. Koch \& W. Oesterreicher have proposed a model of the relations between written and spoken language. After the publication of an important encyclopedic contribution in French (Koch \& Oesterreicher, 2001), this model became very popular in Europe, particularly in the Francosphere. Their paper defends the idea that linguistic variation is the result of polarized social needs of communication: in fact "written language" refers to the manner in which we use language in "communicative distant situation" (like in law texts, but also conferences or pleadings) and the "spoken language" corresponds to language use in situations of social proximity (conversations, emails to close people...). Thus defined, the opposition between written and spoken language are not much linked with the medium, but is socially determined. After briefly presenting this theoretical proposal here, I will suggest two complementary explanations. The first one, about the assimilation of medium and text style ("conception"), explains why it is necessary to distinguish between linguists' and users' points of view. The second one shows why the indubitable relevance of the social perspective on linguistic variation must not lead to the exclusion of another fundamental question for semiology which is: how systems of signs and their uses are determined by their substance (graphic or phonic).

\section{INDEX}

Keywords : Koch, Oesterreicher, written vs spoken language, variation, communication, substance, media, style

Mots-clés : Koch, Oesterreicher, langue orale vs langue écrite, media, substance, variation, communication, style 
AUTEUR

\section{RUDOLF MAHRER}

Université de Lausanne, ch-1015 Lausanne, Suisse 